Randomised controlled trial

\section{Effects of screening and lifestyle counselling on incidence of ischaemic heart disease in general population are limited}

\author{
10.1136/ebmed-2014-110068
}

\section{Scott A Lear}

Faculty of Health Sciences, Simon Fraser University

Division of Cardiology, Providence Health Care

Correspondence to: Dr Scott A Lear, Healthy Heart Program, St. Paul's Hospital, 180-1081 Burrard Street, Vancouver, British Columbia, Canada V5V 3J8; slear@providencehealth.bc.ca

Commentary on: Jorgensen T, Jacobsen RK, Toft U, et al. Effect of screening and lifestyle counselling on incidence of ischaemic heart disease in general population: Inter99 randomised trial. BMJ 2014;348:g3617.

\section{Context}

While mortality rates from cardiovascular diseases-particularly ischaemic heart disease (IHD)-have been declining in Western countries, IHD still contributes a significant burden to individuals, healthcare systems and society. ${ }^{1}$ Therefore, IHD prevention efforts could have major impact on societal health. Given the widespread prevalence of IHD and IHD risk factors, population-focused public health strategies are needed, especially as the value of individual, high-risk programmes will be limited to the individuals directly targeted. The Inter99 randomised trial was designed to test a screening and lifestyle counselling programme to reduce individual-level IHD risk. After 5 years, the Inter99 intervention resulted in significant risk reduction. Jorgensen and colleagues report on a 10-year follow-up of the study to assess the effects on population-level IHD incidence.

\section{Methods}

Between 1999 and 2001, 61301 people aged 30-60 were randomised to either intervention or a control group (at a ratio of roughly 1:4). People in the intervention underwent a comprehensive screening assessment for IHD risk and were grouped into risk categories. Those defined as high risk (upper quintile and/or with overt risk factors) were invited to participate in a personal lifestyle counselling session followed by group lifestyle counselling sessions over a period of up to 6 months. High-risk participants were again assessed at 1 and 3 years, with those still at high risk again offered the group lifestyle counselling sessions. All intervention participants were invited back after 5 years for another assessment and lifestyle planning session. Ten-year follow-up data on death and IHD-related events were obtained using registry data.

\section{Findings}

Of the 11708 patients assigned to the intervention, 52\% attended the baseline assessment. Of these, 3352 were designated high risk and offered group counselling sessions, although less than half (1293) attended. These numbers decreased significantly for attendance at the 1-year and 3-year group counselling sessions. After 10 years, 3163 people had died, 2782 had developed IHD, while 1726 had suffered a stroke. There was no significant difference in primary and secondary outcomes of IHD, stroke and death.

\section{Commentary}

Regular physical activity, a diet that includes a variety of fruits and vegetables and low-fat protein sources, and the abstinence or cessation of smoking are all lifestyle behaviours proven to prevent IHD. For people with IHD, multifactorial cardiac rehabilitation programmes have been proven to reduce death and subsequent IHD through lifestyle management. ${ }^{2}$ However, promoting lifestyle behaviours and reducing IHD at a population level has proven to be more challenging. The current paper further highlights the challenges of lifestyle counselling in reducing IHD in high-risk patients with no overt IHD. The intervention was designed to be a public health screening and IHD prevention programme. While it was successful in improving lifestyle behaviours in those people who participated, it did not alter the overall IHD rate in the intervention group.

While the authors tout the intervention as population focused, it would be more accurate to describe it as being targeted to a high-risk group that represents only a small proportion of the overall population. Indeed, even of the high-risk patients offered counselling sessions only half participated initially, with this proportion decreasing over time, again highlighting the challenges of individualised lifestyle counselling in those without disease. Therefore, only a small number of the intervention population actually received any true intervention and as a result it is not surprising that the authors did not see a wider effect on the population.

Population health interventions must target a wider proportion of people than just those at high risk. As the authors point out, lifestyle counselling is too costly to provide to all individuals, and is only one aspect. Counselling is of little use without the social and community supports available. The literature now reveals the importance of environmental and social policies, which can either facilitate or hinder a healthy lifestyle. ${ }^{3}$ For example, multifaceted government regulation of tobacco has led to greater reductions in smoking rates than any lifestyle counselling programme could ever achieve. Similarly, counselling people to eat health foods when they live in communities that offer fast food restaurants and convenience food stores is going to have little effect. When considering population health interventions we need to move outside of the clinical paradigm and involve a broad range of programmes, policies and practices that support and complement one another-a move already recognised by the United Nations 2011 assembly on non-communicable diseases. $^{5}$

Competing interests None.

Provenance and peer review Commissioned; internally peer reviewed.

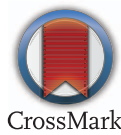

\section{References}

1. World Health Organization. European health report: health and health systems. WHO, 2009.

2. Taylor RS, Brown A, Ebrahim S, et al. Exercise-based rehabilitation for patients with coronary heart disease: systematic review and meta-analysis of randomized controlled trials. Am J Med 2004;116:682-92.

3. Durand CP, Andalib M, Dunton GF, et al. A systematic review of built environment factors related to physical activity and obesity risk: implications for smart growth urban planning. Obes Rev 2011;12:e173-82.

4. Rahmanian E, Gasevic D, Vukmirovich I, et al. The association between the built environment and dietary intake-a systematic review. Asia Pac J Clin Nutr 2014;23:183-96.

5. United Nations. Political declaration of the high-level meeting of the general assembly on the prevention and control of non-communicable diseases. New York: United Nations, 2012. 